\title{
Bogoliubov theory of the Hawking effect in Bose-Einstein condensates
}

\author{
U. Leonhardt ${ }^{1}$, T. Kiss ${ }^{1,2,3}$, and P. Öhberg ${ }^{1,4}$ \\ ${ }^{1}$ School of Physics and Astronomy, University of St Andrews, \\ North Haugh, St Andrews KY16 9SS, Scotland \\ ${ }^{2}$ Research Institute for Solid State Physics and Optics, \\ H-1525 Budapest, P. O. Box 49, Hungary \\ ${ }^{3}$ Institute of Physics, University of Pécs, \\ Ifjúság u. 6. H-7624 Pécs, Hungary \\ ${ }^{4}$ Department of Physics, University of Strathclyde, \\ Glasgow G4 0NG, Scotland
}

\begin{abstract}
Artificial black holes may demonstrate some of the elusive quantum properties of the event horizon, in particular Hawking radiation. One promising candidate is a sonic hole in a Bose-Einstein condensate. We clarify why Hawking radiation emerges from the condensate and how this condensed-matter analog reflects some of the intriguing aspects of quantum black holes.
\end{abstract}

Keywords: Artificial black holes, Bose-Einstein condensates. 


\section{Introduction}

Picture a Bose-Einstein condensate flowing through a nozzle where the condensate exceeds the speed of sound. Suppose that the nozzle is designed such that the transsonic flow does not become turbulent. One could build such a nozzle, the equivalent of the Laval nozzle [1], out of light, using the dipole force between light and atoms to confine the condensate in an appropriate potential. Consider the fate of sound waves propagating against the current of the transsonic condensate. In the subsonic region sound waves may advance against the flow, whereas in the supersonic zone they are simply swept away. No sound can escape the point where the flow turns supersonic - the sonic horizon. The transsonic fluid acts as the acoustic equivalent of the black hole [2, 3].

An artificial black hole [⿴囗十 of this kind could be employed to demonstrate some elusive quantum properties of the event horizon in the laboratory, in particular Hawking radiation [5, 6]. Hawking [7] predicted that the event horizon emits quanta as if the horizon had a temperature given by the gradient of the gravitational potential. To be more precise, the horizon should spontaneously emit quantum pairs where one particle of each pair falls into the hole and the other escapes into space, constituting the radiation of the horizon. Both the spectral distribution and the quantum state of the emerging radiation are thermal. For solar-mass or larger black holes the Hawking temperature is in the order of $10^{-7} \mathrm{~K}$ or below, which makes the effect next to impossible to observe in astronomy. In the case of sonic holes the Hawking temperature is given by the velocity gradient $\alpha$ at the sonic horizon [2, 3],

$$
k_{B} T=\frac{\hbar \alpha}{2 \pi},
$$

and the emitted quanta are phonons. A velocity gradient of $10^{3} \mathrm{~Hz}$ would correspond to about $1.2 \mathrm{nK}$ temperature. In order to observe such subtle quantum effects one should employ the best and coldest superfluids available — Bose-Einstein condensates of dilute gases $[8]$.

Some detailed schemes for sonic black holes in Bose-Einstein condensates have been investigated theoretically [9, 10, 11, 12, 13]. The ultimate design depends on experimental details and on the state of the art in manipulating condensates, a rapidly evolving field. In this paper we analyze the general aspects of the Hawking effect in Bose-Einstein condensates. In the first part of the paper we collect and combine the ingredients of the effect, results that are scattered in the literature. In the second part we show how the Hawking effect arises naturally within the Bogoliubov theory of the elementary excitations in Bose-Einstein condensates [14, 15]. For the first time, to our knowledge, we connect the quantum physics of the event horizon to the behavior of a realistic quantum fluid. 


\section{Sound in fluids}

Consider the propagation of sound in fluids moving with flow speed $\mathbf{u}$. Suppose that the flow varies little over the scale of an acoustic wavelength. In this regime we can describe sound propagation in geometrical acoustics (the equivalent of geometrical optics [16] or of the semiclassical approximation in quantum mechanics [17]). Sound rays follow Hamilton's equations,

$$
\frac{d \mathbf{r}}{d t}=\frac{\partial \omega}{\partial \mathbf{k}}, \quad \frac{d \mathbf{k}}{d t}=-\frac{\partial \omega}{\partial \mathbf{r}},
$$

where the dispersion relation between the frequency $\omega$ and the wave vector $\mathbf{k}$ defines the effective Hamiltonian $\omega(\mathbf{r}, \mathbf{k})$. Assume that in each fluid cell

$$
\omega^{\prime 2}=c^{2} k^{2}
$$

where $c$ denotes the speed of sound and $\omega^{\prime}$ refers to the frequency in locally comoving frames. In the laboratory frame, $\omega^{\prime}$ is Doppler-shifted,

$$
\omega^{\prime}=\omega-\mathbf{u} \cdot \mathbf{k} .
$$

In order to see why waves in fluids are related to waves in general relativity, we write the dispersion relation (3) in a relativistic form. We introduce the space-time wave vector

$$
k_{\nu}=(-\omega, \mathbf{k})
$$

and the matrix

$$
g^{\mu \nu}=\Omega^{-2}\left(\begin{array}{cc}
1 & \mathbf{u} \\
\mathbf{u} & -c^{2} \mathbb{1}+\mathbf{u} \otimes \mathbf{u}
\end{array}\right) .
$$

The prefactor $\Omega$ is an arbitrary non-negative function of the coordinates called the conformal factor. In this notation the dispersion relation appears in the relativistic form

$$
g^{\mu \nu} k_{\mu} k_{\nu}=0,
$$

adopting Einstein's summation convention. Therefore, sound waves experience the moving fluid as an effective space-time geometry with the metric $g_{\mu \nu}$, the inverse matrix of $g^{\mu \nu}$, given by

$$
g_{\mu \nu}=\Omega^{2}\left(\begin{array}{cc}
c^{2}-u^{2} & \mathbf{u} \\
\mathbf{u} & -\mathbb{1}
\end{array}\right) .
$$

The analogy between sound waves in fluids and waves in general relativity turns out to be exact for an irrotational fluid [18] with arbitrary density profile $\rho_{0}$, flow $\mathbf{u}$ and speed of sound $c$, where $\rho_{0}, \mathbf{u}$ and $c$ may vary in space and time. The velocity potential $\varphi$ and the density perturbations $\rho_{s}$ of sound obey the linearized equation of continuity and the linearized Bernoulli equation [19]

$$
\begin{aligned}
\partial_{t} \rho_{s}+\nabla \cdot\left(\mathbf{u} \rho_{s}+\rho_{0} \nabla \varphi\right) & =0 \\
\left(\partial_{t}+\mathbf{u} \cdot \nabla\right) \varphi+c^{2} \frac{\rho_{s}}{\rho_{0}} & =0 .
\end{aligned}
$$


As a consequence, the velocity potential $\varphi$ of sound obeys the equation [3]

$$
\partial_{t} \frac{\rho_{0}}{c^{2}}\left(\partial_{t}+\mathbf{u} \cdot \nabla\right) \varphi+\nabla \cdot \frac{\rho_{0}}{c^{2}}\left[\mathbf{u} \partial_{t}-\left(c^{2}-u^{2}\right)\right] \varphi=0
$$

which can be written as the relativistic wave equation [2, 3]

$$
D_{\nu} D^{\nu} \varphi=\frac{1}{\sqrt{-g}} \partial_{\mu} \sqrt{-g} g^{\mu \nu} \partial_{\nu} \varphi=0
$$

with the conformal factor $\Omega$, in $d$ spatial dimensions, chosen as [3]

$$
\Omega=\left(\frac{\rho_{0}}{c^{3}}\right)^{1-d} .
$$

The assumptions made in order to derive the wave equation (11) are: The fluid [19] is irrotational (1) and isentropic (2). Bose-Einstein condensates naturally satisfy condition (1). Condition (2) characterizes the hydrodynamic regime of condensates [8]. Here the local pressure depends only on the density and on the temperature of the fluid and the quantum pressure is negligible [8]. Condition (2) turns out to be violated close to the sonic horizon.

\section{Sonic horizon}

Consider the propagation of sound waves in the vicinity of the sonic horizon. Focus on the physics in the direction $z$ of the flow at the horizon in a quasi-onedimensional model. Assume that the speed of sound in the fluid is constant. The wave equation (11) reads explicitly

$$
\partial_{t}\left(\partial_{t}+u \partial_{z}\right) \varphi+\partial_{z}\left[u \partial_{t}-\left(c^{2}-u^{2}\right) \partial_{z}\right] \varphi=0
$$

We obtain the general solution

$$
\varphi=\varphi_{0}\left(\tau_{ \pm}-t\right), \quad \tau_{ \pm}=\int \frac{d z}{c \pm u} .
$$

The $\tau_{ \pm}-t$ refer to null coordinates in the frame comoving with the fluid [20]. In these coordinates sound waves propagate exactly like in homogenous space. In the laboratory frame sound waves are accelerated or slowed down by their carrier, the moving fluid. An interesting behavior occurs near the horizon, say at $z=0$, where

$$
u=-c+\alpha z .
$$

The constant $\alpha$ describes the velocity gradient of the condensate at the sonic horizon. We see that

$$
\tau_{+}=\frac{\ln \left(z / z_{\infty}\right)}{\alpha}
$$

Wave packets localized just before the horizon at $z \gtrsim 0$ take an exponentially large time to advance against the current. On the other side of the horizon, $z \lesssim 0$, such 
waves drift equally slowly in the direction of the flow. The horizon at $z=0$ marks a clear watershed, cutting space into two disconnected regions. In terms of the coordinate (17) these regions are characterized by the sign of $z_{\infty}$. For stationary sound waves with frequency $\omega$ we get from the general solution (15)

$$
\varphi=\operatorname{Re}\left\{\varphi_{A} z^{i \omega / \alpha} e^{-i \omega t}\right\} .
$$

The phase of the wave, $(\omega / \alpha) \ln \left(z / z_{\infty}\right)$, diverges logarithmically at the horizon where, in turn, the wavenumber $k$ develops a pole,

$$
k=\frac{\partial}{\partial z}\left(\frac{\omega}{\alpha} \ln \frac{z}{z_{\infty}}\right)=\frac{\omega}{\alpha z},
$$

and the wavelength of sound shrinks beyond all scales,

$$
\lambda=\frac{2 \pi}{k}=\lambda_{0} \alpha z .
$$

However, when $\lambda$ reaches the scale of the healing length $\xi$ of the condensate [8] (also called the correlation length) the hydrodynamic description of sound in BoseEinstein condensates is no longer valid [8]. The acoustic theory at the horizon predicts its own demise. Similarly, waves near the event horizon of a gravitational black hole are compressed beyond all scales. New physics beyond the Planck scale may affect the event horizon [21, 22, 23, 24].

\section{Bogoliubov dispersion}

For Bose-Einstein condensates the equivalent of trans-Planckian physics is wellknown - Bogoliubov's theory of elementary excitations. In the dispersion relation (3) we replace the right-hand side by Bogoliubov's famous result [8, 25, 26, 27]

$$
\omega^{\prime 2}=c^{2} k^{2}\left(1+\frac{k^{2}}{k_{c}^{2}}\right) .
$$

The parameter $k_{c}$ is the acoustic Compton wave number

$$
k_{c}=\frac{m c}{\hbar}=\frac{1}{\xi \sqrt{2}},
$$

with $m$ being the atomic mass, expressed also in terms of the healing length $\xi$ [ (the correlation length). Typically, $\xi$ is in the order of $10^{-6} \mathrm{~m}$ and $c$ reaches a few $10^{-3} \mathrm{~m} / \mathrm{s}$ in Bose-Einstein condensates (without exploiting Feshbach resonances). We calculate the group velocity

$$
\begin{aligned}
v & =\frac{\partial \omega}{\partial k}=u+v^{\prime} \\
v^{\prime} & =\frac{\partial \omega^{\prime}}{\partial k}=c^{2} \frac{k}{\omega^{\prime}}\left(1+\frac{2 k^{2}}{k_{c}^{2}}\right) .
\end{aligned}
$$


Equation (23) shows that the group velocity obeys the Galilean addition theorem of velocities. Equation (24) expresses the group velocity in the fluid frame, $v^{\prime}$, in terms of the frequency and the wavenumber. The acoustic Compton wavenumber, $k_{c}$, sets the scale beyond which $v^{\prime}$ deviates significantly from $c$. For large wavelengths, sound is communicated by atomic collision, and the product of the condensate's density and the atomic collision strength gives $m c^{2}$ [8]. For wavelengths comparable with or shorter than the healing length, the interaction-free Schrödinger dynamics of the atoms dominates the transport of excitations. Perturbations of the free wavefunction travel with infinite velocity. So the acoustic Compton wavenumber, $k_{c}$, characterizes the crossover between the speed of sound and the infinite speed of perturbations of free matter waves.

Close to the sonic horizon, the wavenumber (19) increases dramatically, and, in turn, the effective speed of sound $v^{\prime}$ grows. The horizon, defined as the place where the fluid exceeds the speed of sound, seems to dissolve like a mirage. Nature appears to prevent the existence of an event horizon. However, we show in Sec. 6 that the horizon still exists, but at a less well-defined location and for a particular class of elementary excitations only. As long as $k^{2}$ is much smaller than $k_{c}^{2}$ we get the acoustic relation

$$
k \sim \frac{\omega}{u \pm c} .
$$

For the other extreme, where $k^{2}$ is much larger than $k_{c}^{2}$, one finds 228, 29]

$$
k \sim \pm 2 k_{c} \sqrt{u^{2} / c^{2}-1}+\frac{\omega u}{c^{2}-u^{2}} .
$$

Consider the turning points $z_{0}$, the points where the group velocity of sound (23) vanishes. If the acoustic dispersion relation (3) were universally valid the horizon would be the turning point. Therefore $|z| \lesssim\left|z_{0}\right|$ does indicate the spatial scale of the trans-acoustical range around the horizon, which defines the spatial delocalization of the horizon. To proceed we recall that elementary excitations are small perturbations of the condensate. Their energies $\hbar \omega$ ought to be much smaller than the mean-field energy of the condensate, which is in the order of $m c^{2}$ (with $c$ being the speed of sound). Therefore,

$$
\varepsilon=\frac{\hbar \omega}{m c^{2}}, \quad|\varepsilon| \ll 1
$$

We expand the solution $z_{0}$ of $v=0$ as a power series in $\varepsilon^{1 / 3}$ and find, to leading order, three turning points in the complex plane given by [30]

$$
z_{0}=\frac{c}{\alpha} \frac{3}{2} \sqrt[3]{-1}\left(\frac{\varepsilon}{2}\right)^{2 / 3}
$$

Far away from the horizon we may characterize the four fundamental solutions of the dispersion relation (21) combined with the Doppler shift (4) by their asymptotics (25) or (26). However, close to a turning point geometrical acoustics alone does not provide a good description of wave propagation anymore. The turning points may cause scattering. The connections between elementary excitations across the 
horizon must be examined with care. Extending $z$ to the complex plane represents an elegant way of analyzing this connection. We find in the Appendix that the acoustic relation (19) remains valid on either the upper or the lower half of the complex plane.

\section{$5 \quad$ Bogoliubov modes}

Elementary excitations are perturbations of the condensate, ripples on the macroscopic wave function $\psi_{0}$ of the condensed atoms. The excitations constitute the non-condensed part of the atomic gas. To describe elementary excitations, the total many-body field operator $\hat{\psi}$ of the atoms is split into two components, the condensate with the mean-field wave function $\psi_{0}$ and the non-condensed part. The mean-field wave function comprises the density profile $\rho_{0}$ and the flow $\mathbf{u}$, as

$$
\psi_{0}=\sqrt{\rho_{0}} e^{i S_{0}}, \quad \mathbf{u}=\frac{\hbar}{m} \nabla S_{0} .
$$

The atomic field operator $\hat{\psi}$ is split into the condensate and the non-condensed part according to the relation

$$
\hat{\psi}=\psi_{0}+e^{i S_{0}} \hat{\phi} .
$$

The non-condensed part consists of Bogoliubov modes $u_{\nu}$ and $v_{\nu}$ [8, 14, 15],

$$
\hat{\phi}=\sum_{\nu}\left(u_{\nu} \hat{a}_{\nu}+v_{\nu}^{*} \hat{a}_{\nu}^{\dagger}\right)
$$

The $u_{\nu}$ and $v_{\nu}$ are subject to the Bogoliubov-deGennes equations [8, 14, 15]. If one requires that the Bogoliubov modes satisfy the orthonormality relations

$$
\begin{aligned}
& \int\left(u_{\nu}^{*} u_{\nu^{\prime}}-v_{\nu}^{*} v_{\nu^{\prime}}\right) d z=\delta_{\nu \nu^{\prime}}, \\
& \int\left(u_{\nu} v_{\nu^{\prime}}-v_{\nu} u_{\nu^{\prime}}\right) d z=0,
\end{aligned}
$$

then the $\hat{a}_{\nu}$ and $\hat{a}_{\nu}^{\dagger}$ obey the commutation relations of Bose annihilation and creation operators, as a consequence of the fundamental commutator of atoms with Bose statistics

$$
\left[\hat{\psi}(z), \hat{\psi}^{\dagger}\left(z^{\prime}\right)\right]=\delta\left(z-z^{\prime}\right)
$$

Each pair of $u_{\nu}$ and $v_{\nu}$ characterizes the spatial shape and the evolution of an excitation wave, and the Fock space of the $\hat{a}_{\nu}$ and $\hat{a}_{\nu}^{\dagger}$ spans the state space of the excitation quasiparticles.

To see how the Bogoliubov modes are related to sound waves, we write down the macroscopic wave function of the condensate combined with one of the excitations,

$$
\psi=\psi_{0}+e^{i S_{0}}\left(u_{\nu}+v_{\nu}^{*}\right) .
$$

We represent $\psi$ as

$$
\psi=\sqrt{\rho} e^{i S}, \quad \rho=\rho_{0}+\rho_{s}, \quad S=S_{0}+s,
$$


where $\rho_{s}$ denotes the local density of the sound wave and $s$ is proportional to the velocity potential

$$
\varphi=\frac{\hbar}{m} s
$$

Assuming that $\rho_{s}$ and $s$ are small perturbations we get

$$
u_{\nu}+v_{\nu}^{*}=\sqrt{\rho_{0}}\left(\frac{\rho_{s}}{2 \rho_{0}}+i s\right) .
$$

Assuming further that $u_{\nu}$ and $v_{\nu}$ are stationary waves with frequency $\omega$, we obtain from our solution (18) of the hydrodynamic sound-wave equation and from the linearized Bernoulli equation (10) the Bogoliubov modes

$$
\begin{aligned}
& u_{\nu} \sim A_{\nu}\left(\frac{\omega}{2 \alpha z}+\frac{m c}{\hbar}\right) z^{i \omega / \alpha} e^{-i \omega t}, \\
& v_{\nu} \sim A_{\nu}\left(\frac{\omega}{2 \alpha z}-\frac{m c}{\hbar}\right) z^{i \omega / \alpha} e^{-i \omega t} .
\end{aligned}
$$

These asymptotic expressions are valid as long as the elementary excitations are sound waves with wave number (19). We show in the Appendix that this is the case sufficiently far away from the turning points and on either the upper or the lower half plane. Here the term $\omega /(2 \alpha z)$ in the expressions (39) is always small compared with $m c / \hbar$. For real and positive $z$ we have

$$
(-z)^{i \omega / \alpha}=e^{-( \pm 2 \pi \omega / \alpha)} z^{i \omega / \alpha} .
$$

The \pm sign refers to the two ways in which we may circumvent the trans-acoustic region, on the upper $(+)$ or on the lower $(-)$ half plane. Modes with the acoustic asymptotics (39) throughout the upper half plane are suppressed on the left side of the horizon and for positive frequencies $\omega$ and enhanced for negative $\omega$. Modes with the asymptotics (39) on the lower half plane show the opposite behavior.

\section{$6 \quad$ Negative energy}

Bogoliubov modes are normalized according to the scalar products (32) and (33). Let us calculate the norm of the modes (39) of the sonic hole. The energy parameter (27) is small and so is the extension of the trans-acoustic region around the horizon, measured roughly by the location of the turning points (28). Consequently, we can neglect the trans-acoustic contribution to the normalization integral (32). We approximate the Bogoliubov modes by their asymptotic expressions (39), utilize the relation (40), and get

$$
\begin{aligned}
\int\left(u_{\nu}^{*} u_{\nu^{\prime}}-v_{\nu}^{*} v_{\nu^{\prime}}\right) d z & \sim\left|A_{\nu}\right|^{2}\left(\int_{-\infty}^{0}+\int_{0}^{+\infty}\right) \frac{2 \omega k_{c}}{\alpha z} z^{i\left(\omega^{\prime}-\omega\right) / \alpha} d z \\
& =\left|A_{\nu}\right|^{2}\left(1-e^{-( \pm 2 \pi \omega / \alpha)}\right) 4 \pi \omega k_{c} \delta\left(\omega^{\prime}-\omega\right) .
\end{aligned}
$$

If we choose modes with the asymptotics (139) on the upper half plane the norm is positive and the $u_{\nu}$ and $v_{\nu}$ may serve as proper Bogoliubov modes. We find the 
normalized amplitude

$$
A_{\nu}=\left[\left(1-e^{-2 \pi \omega / \alpha}\right) 4 \pi \omega k_{c}\right]^{-1 / 2} .
$$

Remarkably, the Bogoliubov norm is positive also for modes with negative frequencies. The Bogoliubov-deGennes equations have a well-known symmetry [14, 15]: If the $\left(u_{\nu}, v_{\nu}\right)$ are solutions then the complex-conjugated and interchanged modes, $\left(v_{\nu}^{*}, u_{\nu}^{*}\right)$, are solutions as well. Yet the norm of the conjugate modes is the negative norm of the original $\left(u_{\nu}, v_{\nu}\right)$. In contrast, sonic black holes generate negativefrequency modes with positive norm, which is the unusual feature that gives rise to the acoustic analog of Hawking radiation [6]. We also see how the mentioned symmetry of the Bogoliubov-deGennes equations [14, 15] appears in our case. If we chose the $u_{\nu}$ and $v_{\nu}$ with the asymptotics (39) on the lower half plane we would get negative normalization integrals (41) for both positive and negative frequencies.

The negative frequencies of the positive-norm Bogoliubov modes give rise to negative energies in the Hamiltonian of the elementary excitations,

$$
\hat{H}=\int \hbar \omega\left(\hat{a}_{+}^{\dagger} \hat{a}_{+}-\hat{a}_{-}^{\dagger} \hat{a}_{-}\right) N d \omega .
$$

Here and later the subscripts \pm refer to positive and negative frequencies, respectively, and $N$ denotes the density of modes. We see that there is no natural ground state of the elementary excitations. In practice, of course, the spectrum is limited by the requirement (27) that the energies of the excitations ought to be much smaller in magnitude than the condensate's mean-field energy. We note that the Hamiltonian (43) is invariant under the Bogoliubov transformations

$$
\begin{aligned}
\hat{a}_{ \pm}^{\prime} & =\hat{a}_{ \pm} \cosh \xi-\hat{a}_{\mp}^{\dagger} \sinh \xi, \\
u_{ \pm}^{\prime} & =u_{ \pm} \cosh \xi-v_{\mp}^{*} \sinh \xi, \\
v_{ \pm}^{\prime} & =v_{ \pm} \cosh \xi-u_{\mp}^{*} \sinh \xi .
\end{aligned}
$$

with an arbitrary real parameter $\xi$. In the case we choose

$$
\tanh \xi=e^{-\pi \omega / \alpha}
$$

we get a new set of modes, Eq. (39), with

$$
A_{ \pm}^{\prime} \sim \Theta( \pm z)\left(4 \pi \omega k_{c}\right)^{-1 / 2} .
$$

The $\Theta$ function indicates that the primed modes appear on either the left or on the right side of the trans-acoustic region. Therefore, despite the trans-Planckian problem, a sonic horizon exists, but at a less well-defined location, within $|z| \lesssim\left|z_{0}\right|$, and the horizon applies to a particular set of modes only. 


\section{Hawking effect}

The Bogoliubov transformations (44) relate one set of quasiparticles to another one, both representing perfectly valid energy eigenvalues, yet their quasiparticle vacua differ (the states $|0\rangle$ or $\left|0^{\prime}\right\rangle$ that are annihilated by $\hat{a}_{ \pm}$and $\hat{a}_{ \pm}^{\prime}$, respectively). This ambiguity has direct physical consequences, because the cloud of non-condensed atoms [26] depends on the vacuum state of the elementary excitations,

$$
\begin{aligned}
\left\langle 0\left|\hat{\phi}^{\dagger} \hat{\phi}\right| 0\right\rangle & =\int\left(\left|v_{+}\right|^{2}+\left|v_{-}\right|^{2}\right) N d \omega \\
\left\langle 0^{\prime}\left|\hat{\phi}^{\dagger} \hat{\phi}\right| 0^{\prime}\right\rangle & =\int\left(\left|v_{+}^{\prime}\right|^{2}+\left|v_{-}^{\prime}\right|^{2}\right) N d \omega \\
& \neq\left\langle 0\left|\hat{\phi}^{\dagger} \hat{\phi}\right| 0\right\rangle .
\end{aligned}
$$

In general relativity the notion of the vacuum is observer-dependent. For example, the vacuum of empty space in Minkowski coordinates appears as a thermal field to accelerated observers [5, 6, 31]. In the case of the black hole, the gravitational collapse has created a state of quantum fields that an inward-falling observer perceives as vacuum, yet an external observer sees as thermal radiation, Hawking radiation [5, 6, 7]. In our case, the equivalent of the gravitational collapse, the formation of the sonic horizon, chooses the quasiparticle vacuum, if the trans-sonic velocity pro-

file has initially been created from a condensate without a horizon. Such a process must be sufficiently smooth to keep the condensate intact.

To analyze the quasiparticle vacuum, we use the Heisenberg picture of quantum mechanics where observables evolve while the quantum state is invariant. We describe the initial (and final) vacuum state with respect to one set of continuous modes given before the formation of the horizon. In the Heisenberg picture these modes evolve. We sort the initial modes into left- and right-moving modes that, close to complex $\infty$, are analytic on the upper or on the lower half plane, respectively, because here $\exp (i k z)$ converges for positive $k$ on the upper and for negative $k$ on the lower half plane. The upstream modes we are interested in stem from right-moving modes. The formation of the sonic horizon, a smooth process, cannot fundamentally alter the analyticity of the vacuum modes. In particular, the process can never create non-analytic modes of the type expressed in Eq. (46). Consequently, the initial quasiparticle vacuum assumes the analytic modes of Eqs. (39) and (42).

Given this vacuum state, we determine the quantum depletion of the condensate. We write the density of the non-condensed atoms in terms of the primed Bogoliubov modes. As we have seen, these modes describe the set of elementary excitations that exhibit the sonic horizon. We find that

$$
\left\langle 0\left|\hat{\phi}^{\dagger} \hat{\phi}\right| 0\right\rangle=\rho(|z|), \quad \rho(z)=\int\left[\left(\left|u_{+}^{\prime}\right|^{2}+\left|v_{+}^{\prime}\right|^{2}\right) \bar{n}(\omega)+\left|v_{+}^{\prime}\right|^{2}\right] N d \omega .
$$

Here $\bar{n}(\omega)$ denotes the average number of non-condensed atoms per excitation mode,

$$
\bar{n}(\omega)=\frac{1}{e^{2 \pi \omega / \alpha}-1}=\frac{1}{e^{\hbar \omega / k_{B} T}-1} .
$$


The non-condensed atoms are Planck-distributed with the Hawking temperature (11). Therefore, as soon as the condensate flows through the nozzle, breaking the speed of sound, a thermal cloud of atoms is formed. This effect is the signature of Hawking radiation for sonic holes in Bose-Einstein condensates. The thermal cloud due to the Hawking effect should be observable when the initial temperature of the atoms is below the Hawking temperature. On the other hand, one could also regard the Hawking effect in the condensate as the quantum depletion (47) of atoms at zero temperature with respect to the analytic modes (42) that transcend the horizon. This feature reflects the ambiguity of the vacuum in general relativity. Using techniques for measuring the population of Bogoliubov modes [32, 33], one could perhaps demonstrate the ambiguity of the vacuum in the laboratory.

Finally we note that within our model sonic black holes are stable, provided of course that the transsonic flow is not plagued by hydrodynamic instabilities. In reality, elementary excitations interact with each other, giving rise to what is known as Landau-Beliaev damping [34, 35, 36]. Since a sonic horizon does not have a ground state, this damping mechanism will lead to the gradual evaporation of the condensate. Therefore, Landau-Beliaev damping [34, 35, 36] plays the role of black-hole evaporation. It is tempting to turn matters around and to approach cosmological problems from the perspective of condensed-matter physics [37, 38, 39].

\section{Acknowledgements}

We thank M. V. Berry, I. A. Brown, L. J. Garay, T. A. Jacobson, R. Parentani, and G. E. Volovik for discussions. Our work was supported by the ESF Programme Cosmology in the Laboratory, the Leverhulme Trust, the National Science Foundation of Hungary (contract No. F032346), the Marie Curie Programme of the European Commission, the Royal Society of Edinburgh, and by the Engineering and Physical Sciences Research Council. 


\section{Appendix}

In this Appendix we examine the asymptotics of the Bogoliubov modes on the complex plane. We use the semiclassical approximation [30, 40]

$$
\begin{aligned}
& u_{\nu}=U_{\nu} \exp \left(i \int k d z-i \omega t\right), \\
& v_{\nu}=V_{\nu} \exp \left(i \int k d z-i \omega t\right) .
\end{aligned}
$$

The wavenumber $k$ should obey Bogoliubov's dispersion relation (21) including the Doppler shift (四). We obtain four fundamental solutions of this fourth-order equation. Figure 2 shows the three branches of $k$ that are relevant in our analysis. The amplitudes $U_{\nu}$ and $V_{\nu}$ obey the relation [30, 40]

$$
\partial_{z}\left(U_{\nu}^{2}-V_{\nu}^{2}\right) v=0
$$

where $v$ denotes the group velocity (23) of the elementary excitation. Equation (52) formulates the conservation law of the quasiparticle flux for stationary states [40] if $z$ is real, where $U_{\nu}^{2}-V_{\nu}^{2}$ gives $\left|U_{\nu}\right|^{2}-\left|V_{\nu}\right|^{2}$ up to a constant phase factor. The relation (52) can be extended to the complex $z$ plane and to complex frequencies $\omega$ [30]. Equation (52) implies that the amplitudes $U_{\nu}$ and $V_{\nu}$ diverge close to a turning point $z_{0}$ where $v$ vanishes. Consequently, the semiclassical approximation breaks down at the turning point [17]. The turning point causes significant scattering, i.e. the conversion of one mode with a given $k$ into two modes, one with wavenumber $k$ and the other one with a different wavenumber that satisfies the dispersion relation as well. At the turning point the two branches coincide. To prove this, we regard for a moment $z$ as a function of $k$ at constant frequencies $\omega=\omega_{0}$, defined implicitly by Eqs. (14), (16) and (21). We get

$$
v=\frac{\partial \omega}{\partial k}=u+\frac{\partial}{\partial k}\left(\omega_{0}-u k\right)=-\alpha k \frac{\partial z}{\partial k} .
$$

We see that the function $z(k)$ reaches extrema at the point $z_{0}$ where $v$ vanishes, i.e. at the turning point. Close to the $z_{0}$ we find after some algebra [30], by expanding $z$ into a power series in $\varepsilon^{1 / 3}$,

$$
z-z_{0} \sim-\frac{3\left(k-k_{0}\right)^{2}}{8 \alpha}, \quad k_{0}=\sqrt[3]{-4 \varepsilon}
$$

to leading order. Consequently, each turning point connects two branches of the wavenumber $k$. In general the mode conversion occurs near specific lines in the complex plane, called Stokes lines in the mathematical literature 41]. Stokes lines, originating from the turning point $z_{0}$, are defined as the lines where the differences between the phases $\int k d z$ of the two connected $k$ branches is purely imaginary. Here one of the waves is exponentially small compared with the other. We obtain from Eq. (54) that the difference between the two branches is proportional to the square root of $z-z_{0}$. Consequently, the phase difference is proportional to $\left(z-z_{0}\right)^{3 / 2}$, 
giving rise to three Stokes lines from each turning point $z_{0}$, as in the traditional case of Schrödinger waves in one dimension [17, 42]. Figure 3 shows the Stokes lines for the three turning points close to the horizon and for the branch cuts of $k$ chosen in Fig. 2.

At a Stokes line the phase difference between the two connected branches is purely imaginary. One of the waves exponentially exceeds the other and, within the semiclassical approximation, the smaller wave is totally overshadowed by the larger one, if the larger wave is present. In general, the Bogoliubov modes consist of a superposition of the four fundamental solutions that correspond to the four branches of the dispersion relation

$$
\begin{aligned}
& u_{\nu}=c_{A} u_{A}+c_{B} u_{B}+c_{C} u_{C}+c_{D} u_{D}, \\
& v_{\nu}=c_{A} v_{A}+c_{B} v_{B}+c_{C} v_{C}+c_{D} v_{D} .
\end{aligned}
$$

The $u_{A}$ and $v_{A}$ refer to the $k$ branch where $k$ obeys the asymptotics (19), i.e. where the wavenumber satisfies the dispersion relation (3) of sounds in moving fluids, taking into account the Doppler detuning (四) and where $k$ corresponds to an upstream wave. We call such Bogoliubov modes acoustic modes. When crossing a Stokes line, the exponentially suppressed solution may gain an additional component that is proportional to the coefficient of the exponentially enhanced solution [42]. If we wish to construct Bogoliubov modes where only the exponentially smaller component exits in the vicinity of a Stokes line we must put the coefficient of the larger one to zero. In Fig. 3 the pairs of letters indicate which branches are connected by the lines, and the first letter identifies the exponentially dominant branch. The picture shows that with the choice of branch cuts made we can construct a Bogoliubov mode that is acoustic on the upper half plane. Trans-acoustic physics is confined to the lower half plane. On the other hand, if we chose other branch cuts of $k$ we may get Bogoliubov modes that are acoustic on the lower half plane and trans-acoustic on the upper one. Therefore, according to Eq. (40), the choice of the $k$ branch determines whether a Bogoliubov mode is larger or smaller beyond the sonic horizon at the real axis, for $z<0$. Branch cuts of $k$ are fairly arbitrary. Given the Bogoliubov mode on the right side of the horizon, we cannot predict within the semiclassical approximation the amplitude of the mode on the left side. Therefore, the two sides are causally disconnected. Within the semiclassical approximation, the horizon is a genuine horizon, despite the acoustic analog of the notorious trans-Planckian problem [21, 22]. 


\section{References}

[1] R. Courant and K. O. Friedrichs, Supersonic Flow and Shock Waves (Wiley, New York, 1967).

[2] W. G. Unruh, Phys. Rev. Lett. 46, 1351 (1981).

[3] M. Visser, Class. Quant. Grav. 15, 1767 (1998).

[4] M. Novello, M. Visser, and G. E. Volovik (editors), Artificial Black Holes (World Scientific, Singapore, 2002).

[5] N. D. Birrell and P. C. W. Davies, Quantum Fields in Curved Space (Cambridge University Press, Cambridge, 1982).

[6] R. Brout, S. Massar, R. Parentani, and Ph. Spindel, Phys. Rep. 260, 329 (1995).

[7] S. M. Hawking, Nature (London) 248, 30 (1974); Commun. Math. Phys. 42, 199 (1975).

[8] F. Dalfovo, S. Giorgini, L. P. Pitaevskii, and S. Stringari, Rev. Mod. Phys. 71, 463 (1999).

[9] L. J. Garay, J. R. Anglin, J. I. Cirac, and P. Zoller, Phys. Rev. Lett. 85, 4643 (2000).

[10] L. J. Garay, J. R. Anglin, J. I. Cirac, and P. Zoller, Phys. Rev. A 63, 023611 (2001).

[11] C. Barcelo, S. Liberati, and M. Visser, Class. Quant. Grav. 18, 1137 (2001).

[12] C. Barcelo, S. Liberati, and M. Visser, arXiv:gr-qc/0110036.

[13] M. Sakagami and A. Ohashi, Prog. Theor. Phys. 107, 1267 (2002).

[14] A. L. Fetter, Ann. Phys. (N.Y.) 70, 67 (1972).

[15] A. L. Fetter, Phys. Rev. A 53, 4245 (1996).

[16] M. Born and E. Wolf, Principles of Optics (Cambridge University Press, Cambridge, 1999).

[17] L. D. Landau and E. M. Lifshitz, Quantum Mechanics (Pergamon, Oxford, 1977).

[18] For a wave equation of sound in fluids with vorticity see S. E. P. Bergliaffa, K. Hibberd, M. Stone, and M. Visser, cond-mat/0106255.

[19] L. D. Landau and E. M. Lifshitz, Fluid Mechanics (Pergamon, Oxford, 1982).

[20] In a fluid extending in more than one spatial dimensions the acoustic space-time geometry in curved, in general. There is no universal comoving frame anymore, because in this frame the metric is flat. 
[21] G. t'Hooft, Nucl. Phys. B 256, 727 (1985).

[22] T. Jacobson, Phys. Rev. D 44, 1731 (1991).

[23] W. G. Unruh Phys. Rev. D 51, 2827 (1995).

[24] R. Brout, S. Massar, R. Parentani, and Ph. Spindel, Phys. Rev. D 52, 4559 (1995).

[25] N. Boboliubov, J. Phys. (Moscow) 11, 23 (1947).

[26] L. D. Landau and E. M. Lifshitz, Statistical Physics. Part 2, Theory of the Condensed State (Pergamon, Oxford, 1980).

[27] Corley and Jacobson studied the effect of a Bogoliubov-type dispersion on scalar waves in Refs. [28, 29].

[28] S. Corley, Phys. Rev. D 57, 6280 (1998).

[29] S. Corley and T. Jacobson, Phys. Rev. D 59, 124011 (1999).

[30] U. Leonhardt, T. Kiss, and P. Öhberg, arXiv:cond-mat/0211462.

[31] W. G. Unruh, Phys. Rev. D 14, 870 (1976).

[32] J. M. Vogels, K. Xu, C. Raman, J. R. Abo-Shaeer, and W. Ketterle, Phys. Rev. Lett. 88, 060402 (2002).

[33] R. Ozeri, J. Steinhauer, N. Katz, and N. Davidson, Phys. Rev. Lett. 88, 220401 (2002).

[34] L. P. Pitaevskii and S. Stringari, Phys. Lett. A 235, 398 (1997).

[35] S. Giorgini, Phys. Rev. A 57, 2949 (1998).

[36] N. Katz, J. Steinhauer, R. Ozeri, and N. Davidson, Phys. Rev. Lett. 89, 220401 (2002).

[37] G. Chapline, E. Hohlfeld, R. B. Laughlin, and D. I. Santiago, Phil. Mag. B 81, 235 (2001).

[38] G. E. Volovik, Phys. Rept. 351, 195 (2001).

[39] S.-C. Zhang and J. Hu, Science 294, 823 (2001).

[40] A. Csordás, R. Graham, and P. Szépfalusy, Phys. Rev. A 57, 4669 (1998).

[41] M. J. Ablowitz and A. S. Fokas, Complex Variables (Cambridge University Press, Cambridge 1997).

[42] W. H. Furry, Phys. Rev. 71, 360 (1947). 


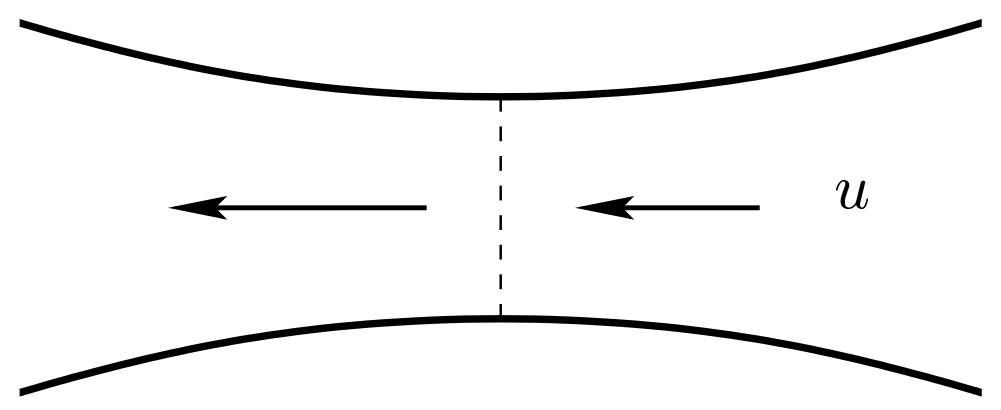

Figure 1: Schematic diagram of a sonic horizon. A fluid is forced to move through a constriction where the flow speed $u$ becomes supersonic (dashed line). The constriction may be formed by the walls of a tube or, if the fluid is an alkali Bose-Einstein condensate, by a suitable trapping potential. The picture shows a Laval nozzle [1] where a supersonic fluid is hydrodynamically stable. 

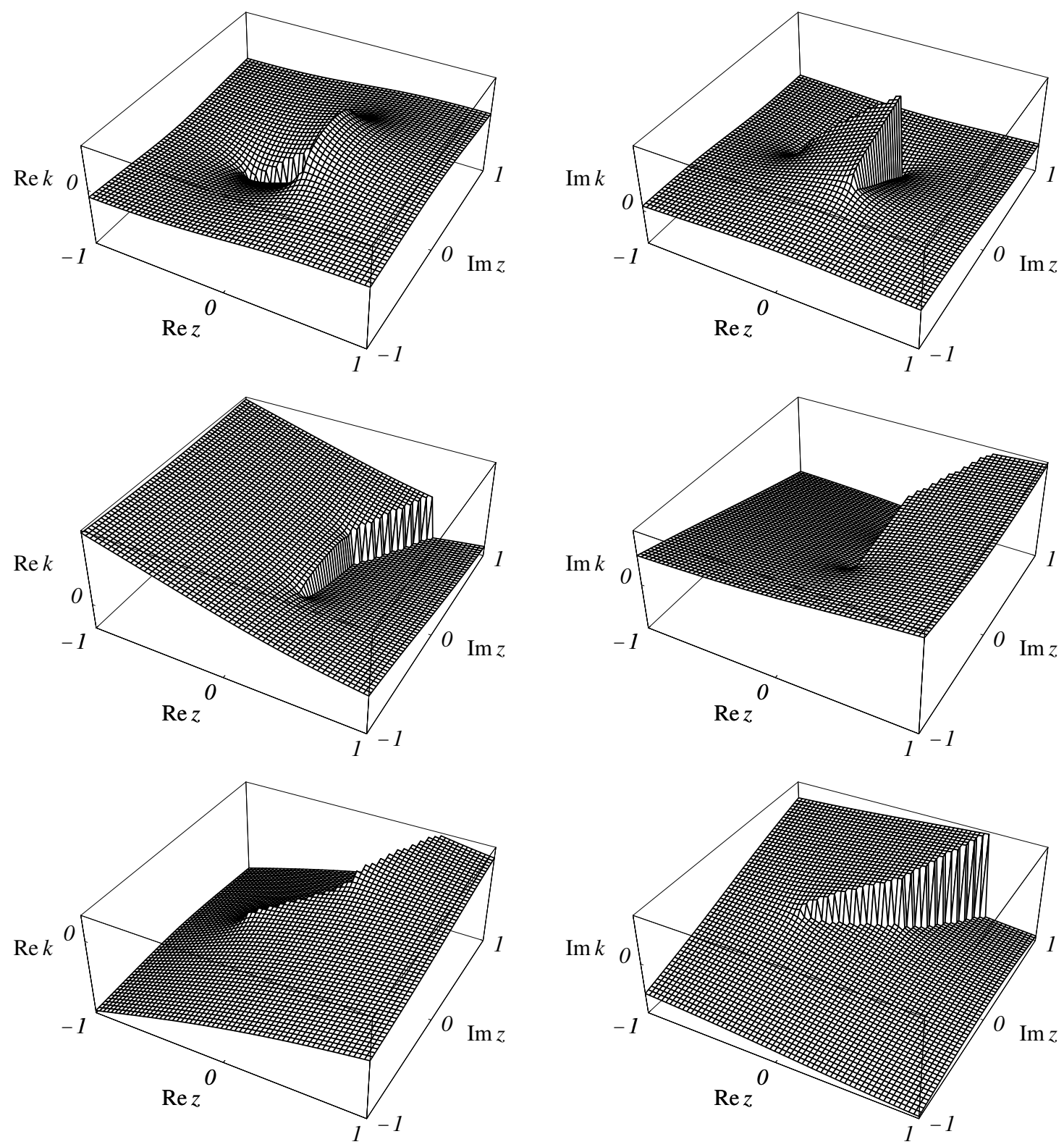

Figure 2: Wavenumbers $k$ of elementary excitations around a sonic black-hole horizon, analytically continued onto the complex plane. The figure shows three roots of the dispersion relation $\left[\hbar^{2} k^{2} /(2 m)+m c^{2}\right]^{2}-\hbar^{2}[\omega-(-c+\alpha z) k]^{2}=m^{2} c^{4}$ for $\omega=0.1\left(m c^{2} / \hbar\right)$ and $\alpha=0.5\left(m c^{2} / \hbar\right)$, illustrating the branch cuts of $k$. The top row displays the wavenumber of a sound wave that propagates against the current. The picture indicates the characteristic $\omega /(\alpha z)$ asymptotics away from the branch points. The two lower rows display two trans-acoustic branches of $k$. The fourth root of the dispersion relation is not shown, because it corresponds to the trivial case of sound waves that propagate with the flow. 


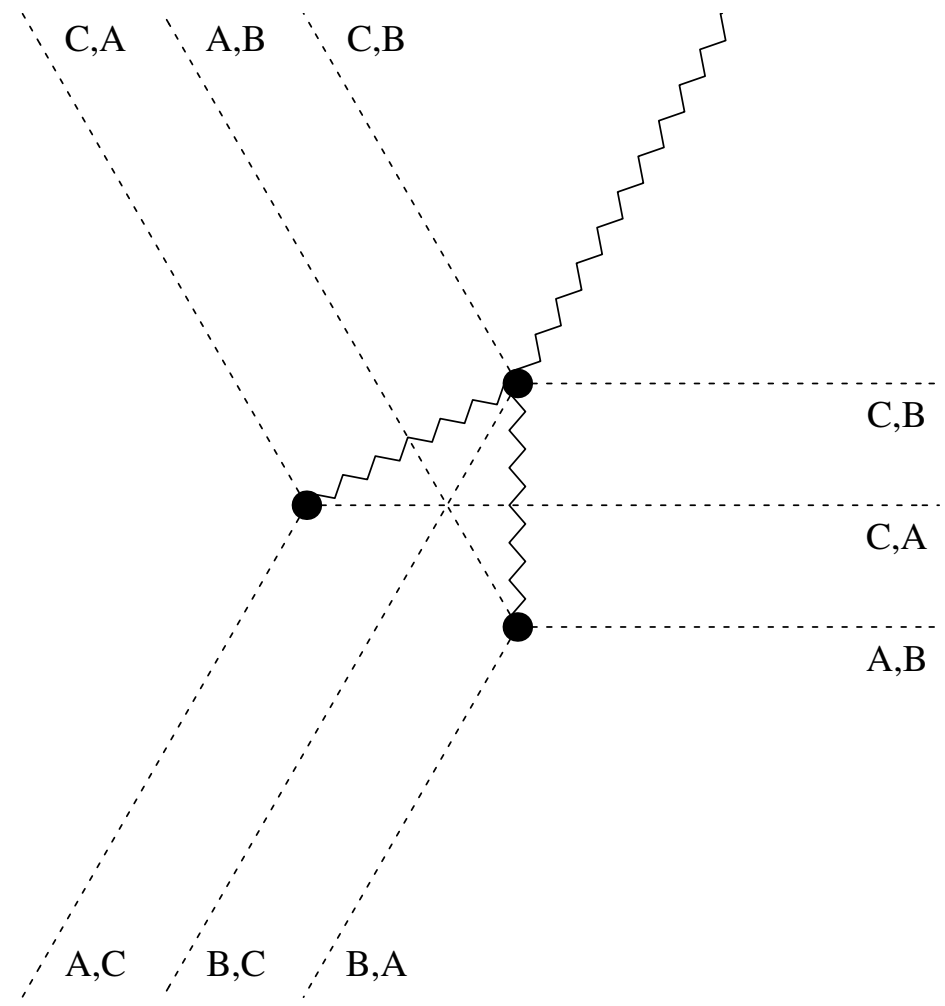

Figure 3: Stokes lines of elementary excitations at a sonic black hole (dotted lines), given the choice of branch cuts made in Fig. 2. The pairs of letters indicate which branches of the superposition (52) are connected by the lines. The first letter of each pair identifies the exponentially dominant branch. We construct a Bogoliubov mode that is acoustic (component $\mathrm{A}$ ) on the upper half plane by putting the coefficient $c_{C}$ to zero on the C,A Stokes line originating from the left turning point. 